\section{Fatores associados ao aleitamento materno nas diferentes Regiões do Brasil}

\section{Factors associated with maternal breastfeeding in different Regions of Brazil}

Daniela Wenzel 1

Sonia Buongermino de Souza 2
1 Departamento de Alimentação Escolar. Secretaria Municipal de Educação. Rua Líbero Badaró, 425. $9^{\circ}$ andar. Centro. São Paulo, SP, Brasil. CEP: 01.009 905. E-mail: danielawenzel@gmail.com 2 Departamento de Nutrição. Faculdade de Saúde Pública. Universidade de São Paulo. São Paulo, SP, Brasil

\begin{abstract}
Objectives: to identify factors associated with maternal breastfeeding in different regions of the country.

Methods: the data form part of the Brazilian 2002-2003 Family Budget Study. The sample comprised 2958 children aged between 0 and 1 year, representative of the national population. And linear regression model with a binomial family and logarithmic connection was created for each region, with a confidence interval of $90 \%$.

Results: an increase in the number of people living in a household is the principal negative factor for breastfeeding in all regions of Brazil. In this sample, negative factors were found to be: mothers of advanced age, having four or more people living in the household, higher income, higher level of education of the mother and the use of a crèche. Positive factors were having two or more children at home under the age of five years and being black or of mixed race.

Conclusions: the regions that had the highest number of negative factors associated with maternal breastfeeding were the Southeast and the South, with a large number of household members being the worst situation for encouraging breastfeeding in all regions of Brazil.
\end{abstract}

Key words Breast feeding, Epidemiologic factors, Geographic mapping

\section{Resumo}

Objetivos: identificar os fatores que determinam o aleitamento materno nas diferentes regiões do país.

Métodos: os dados fazem parte da Pesquisa de Orçamento Familiar de 2002-2003 realizada no Brasil. A amostra foi de 2958 crianças de zero a um ano, representativas da população nacional. Um modelo de regressão linear (GLM) com família binomial e ligação logarítmica foi criado para cada região, com um intervalo de confiança de $90 \%$.

Resultados: o aumento do número de moradores no domicilio é o principal fator negativo para a amamentação em todas as regiões do Brasil. Foram considerados como fatores desfavoráveis, no conjunto da amostra: mães com idade avançada, ter quatro ou mais moradores no domicílio, maior renda, maior escolaridade materna e uso de creche. Os fatores favoráveis foram: ter dois ou mais filhos menores de cinco anos em casa e mães de cor preta ou parda.

Conclusões: as regiões que apresentaram um número maior de fatores desfavoráveis ao aleitamento materno foram Sudeste e Sul, sendo que o maior número de moradores no domicílio foi a pior situação para a efetivação da amamentação nas regiões do Brasil.

Palavras-chave Aleitamento materno, Fatores epidemiológicos, Mapeamento geográfico 


\section{Introdução}

Estudos científicos são unânimes em mostrar os benefícios da amamentação para a saúde infantil. A superioridade do leite humano como fonte de nutrientes, proteção contra doenças e laços de afeto entre mães e filhos, faz com que especialistas de todo o mundo o recomendem. ${ }^{1-3}$

O panorama mundial da amamentação mostra grande heterogeneidade em relação à prevalência do aleitamento materno, não só entre países, mas também entre regiões de um mesmo país. Grande parte da literatura sobre a extensão da prática da amamentação fornece informações sobre cidades e áreas muito específicas, e poucos mostram dados sobre a situação geral de um país. Por exemplo, nas últimas duas décadas em estudo na região norte da Rússia, a taxa de aleitamento materno foi de $47,2 \%{ }^{4}$ $\mathrm{Na}$ região noroeste da China, a prevalência foi de $75 \%$ e este valor foi semelhante às décadas anteriores. ${ }^{5} \mathrm{Na}$ Itália, na região sul a prevalência do aleitamento materno foi de $30 \%$ e $41 \%$ na Região Norte. 6

A partir da década de 1980, várias estratégias têm sido propostas e implementadas diversas campanhas a fim de ampliar a prática do aleitamento materno no Brasil7 e em diferentes regiões do país. ${ }^{8}$ $\mathrm{Na}$ década de 2000, aumento nos valores da prevalência do aleitamento materno foi observado principalmente na Região Norte que apresentou taxa de $62 \%$ para crianças até seis meses e $44 \%$ para maiores de seis meses. ${ }^{9}$ A Pesquisa Nacional de Demografia e Saúde da Mulher e da Criança (PNDS), 10 realizada em 2006 em todo o país, mostra que a frequência de aleitamento materno predominante e complementar ocorreu, respectivamente, em $8,2 \%$ e $62,4 \%$ das crianças de 4 a 6 meses. Em 2009, na II Pesquisa de Prevalência de Aleitamento Materno nas Capitais Brasileiras e Distrito Federal também foi verificado crescimento da freqüência do aleitamento em todas as regiões do país, principalmente na Região Norte com 76,91\% de prevalência. ${ }^{11}$

É inquestionável a importância do conhecimento dos determinantes do aleitamento materno. Embora os estudos não sejam conclusivos, alguns fatores sociodemográficos e culturais parecem afetar, direta ou indiretamente, a situação do aleitamento materno, como: a idade materna, verificando-se maior probabilidade de amamentação em mães mais jovens em relação às mais velhas. ${ }^{11}$ Quanto à etnia, mães brancas amamentam menos seus filhos em comparação com as mães pretas e pardas.11,12 Em relação ao nível social, tem sido observado que as mães com menor escolaridade e baixos rendimentos amamentam com mais frequência do que as mães de nível superior, 13 embora venha ocorrendo uma tendência crescente da amamentação entre mulheres mais escolarizadas, com maior acesso à informação, e melhor situação econômica. ${ }^{14-16}$

No Brasil existe uma importante diversidade sócio-cultural e territorial. Seu território está dividido em cinco macrorregiões e todas se dividem em áreas urbanas e rurais. $\mathrm{O}$ conhecimento sobre as tendências regionais dos determinantes do aleitamento materno é de fundamental importância, pois permite avaliar e explicar as mudanças e promover ajustes nas práticas de promoção e incentivo ao aleitamento materno, segundo situações regionais. Neste contexto, o presente estudo tem como objetivo identificar os fatores associados ao aleitamento materno nas diferentes regiões do país.

\section{Métodos}

Trata-se de um estudo transversal, em que os dados apresentados fazem parte da Pesquisa de Orçamentos Familiares (POF), realizado pelo Instituto Brasileiro de Geografia e Estatística (IBGE), em 2002-2003, em todas as regiões do Brasil. A POF foi composta por uma amostra de 48.470 famílias. A estratificação geográfica permitiu a representatividade de todo o território nacional (Regiões Norte, Nordeste, Sudeste, Sul e CentroOeste), além de áreas urbanas e rurais. A amostra foi formada por conglomerados em dois estágios, com estratificação estatística e geográfica. A estratificação da amostra foi definida em cada estrato geográfico, a partir de variável que caracteriza os estratos socioeconômicos. 17 Optou-se por selecionar em cada setor um número maior de famílias para compor a amostra, estipulando uma proporção de $25 \%$ para compensar a não-resposta. A amostragem foi descrita em publicação do IBGE de 2004. ${ }^{17}$

A coleta de dados foi realizada entre 2002 e 2003. Os dados foram obtidos diretamente nos domicílios selecionados por aplicação de questionário por meio de entrevistas com membros da família.

Para este estudo, foi composta uma amostra de 2958 crianças de zero a um ano de idade, que representavam a população nacional nessa faixa etária. Extraíram-se duas subamostras, uma com 1477 crianças de 0 a 180 dias (grupo 1) e outra de 1481 crianças de 181 a 365 dias (grupo 2). Na POF procurou-se avaliar a família como um todo, portanto, não foram incluídas nos questionários perguntas específicas sobre amamentação. No entanto, na questão sexo foram atribuídas às 
mulheres as categorias gestante e lactante, o que tornou possível obter a informação sobre a ocorrência do aleitamento materno na população investigada, uma vez que, com a categoria lactante, foi possível selecionar as mulheres que estavam amamentando e cruzar esta variável com a idade da criança, selecionando apenas as menores de um ano de idade, foco deste estudo.

A variável dependente, aleitamento materno (AM), foi categorizada crianças não-amamentadas e crianças amamentadas. O AM foi definido como a oferta de leite de peito, independente de as crianças estarem ou não recebendo outro tipo de alimento líquido ou sólido, ou qualquer outro tipo de leite.

As variáveis independentes foram: idade da mãe $(<21,21-25,26-30,>30$ anos $)$, escolaridade materna ( $\leq 4$ anos, $5-8$ anos e $\geq 9$ anos de estudos), número de moradores no domicílio (1-3, 4-5, +6 moradores), número de crianças menores de cinco anos no domicilio ( 1 criança, 2 crianças , 3 ou mais crianças), etnia (branca, preta e parda), Regiões (Norte, Nordeste, Sudeste, Sul e Centro-Oeste), renda domiciliar per capita (quartis de renda), frequência em creche (sim e não) e tipo de área (rural e urbana).

As diferenças entre as regiões na distribuição dos determinantes do aleitamento materno foram analisadas em tabelas de contingência e as associações testadas pelo teste qui-quadrado, com nível de significância de 5\%.

Para a análise da presença de variações nos determinantes do AM entre as regiões do país, utilizou-se um modelo hierárquico de dois níveis: $\mathrm{O}$ nível distal, composto pelas variáveis área, número de moradores no mesmo domicílio, renda e o nível proximal, composto pelas variáveis crianças menores de cinco anos, etnia, uso de creche, escolaridade materna e idade materna . Construiu-se um modelo de regressão linear (GLM) com família binomial e ligação logarítmica para cada região. A magnitude da associação foi estimada pelo cálculo da Razão de Prevalência bruta e ajustada, com seus respectivos intervalos de confiança de $90 \%$. A regressão de Poisson com variância robusta foi utilizada no caso de não convergência.

A análise dos dados foi realizada utilizando o programa STATA, versão 11. O estudo foi aprovado pelo Comitê de Ética em Pesquisa da Faculdade de Saúde Pública da Universidade de São Paulo.

\section{Resultados}

As características da população de estudo nas Regiões do Brasil são descritas na Tabela 1. Todas as regiões têm maior proporção de pessoas vivendo em áreas urbanas. Nas Regiões Norte e Nordeste, a maior parte das mães é mais jovem, com menor nível de escolaridade e baixo nível de renda. $\mathrm{Na}$ Região Centro-Oeste, a maior parte das mães são jovens, porém com maior nível de escolaridade e de renda. Diferentemente do restante das Regiões, na Região Sudeste a maior parte da população de mães tinha maior escolaridade, idade mais elevada e com maior renda.

A associação entre o AM com fatores sociais, demográficos e econômicos segundo as macrorregiões do Brasil nos grupos etários de 0 a 180 dias e de 181 a 365 dias são apresentadas nas Tabelas 2 e 3 , respectivamente.

Verificou-se que o maior número de moradores no domicilio é o principal fator negativamente associado à amamentação nos dois grupos etários nas regiões do Brasil.

$\mathrm{Na}$ Região Norte não houve associação do AM com os fatores estudados e no grupo de 0 a 180 dias, enquanto entre as crianças com idade entre $181 \mathrm{e}$ 365 , residir em domicílios com maior número de pessoas esteve negativamente associado à amamentação. Nessa mesma região, residir em área rural esteve positivamente associado à amamentação.

$\mathrm{Na}$ Região Nordeste, o AM esteve negativamente associado ao maior número de moradores no domicílio nos dois grupos etários, enquanto que idade materna esteve associada ao AM no grupo de 181 a 365 dias. Na Região Centro-Oeste, no grupo com idade de 0 a 180 dias, maior renda foi um fator positivamente associado ao AM. Entre as crianças com idade entre 181 e 365 anos, viver em domicílios com maior número de crianças menores de cinco anos aumentou a chance de amamentação

No Sudeste, entre as crianças com idade entre 0 e 180 dias, frequentar creche foi um fator negativamente associado ao AM. Entre as crianças dessa região, com idade superior a 180 dias, maior número de crianças menores de cinco anos na residência e ser filho de mãe de cor preta ou parda estiveram positivamente associados ao AM, enquanto que o maior nível de escolaridade materna se associou negativamente à amamentação. Na Região Sul, entre as crianças menores do que 180 dias, maior número de moradores no domicílio, maior renda e maior idade materna estiveram negativamente associados ao AM. Entre as crianças maiores de 180 dias, maior número de moradores na residência e maior renda estiveram negativamente associados, enquanto que ser filho de mãe preta ou parda aumentou a chance da amamentação. 
Tabela 1

Características das variáveis independentes nas regiões do Brasil, 2002 -2003.

\begin{tabular}{|c|c|c|c|c|c|c|c|c|c|c|c|}
\hline \multirow[t]{3}{*}{ Variável } & \multicolumn{10}{|c|}{ Macrorregiões do Brasil } & \multirow[t]{3}{*}{$p$} \\
\hline & \multicolumn{2}{|c|}{ Norte } & \multicolumn{2}{|c|}{ Nordeste } & \multicolumn{2}{|c|}{ Sudeste } & \multicolumn{2}{|c|}{ Sul } & \multicolumn{2}{|c|}{ Centro-Oeste } & \\
\hline & $\mathbf{n}$ & $\%$ & $\mathbf{n}$ & $\%$ & $\mathbf{n}$ & $\%$ & $\mathbf{n}$ & $\%$ & $\mathbf{n}$ & $\%$ & \\
\hline Área & & & & & & & & & & & $<0,001$ \\
\hline Rural & 1065 & 36,0 & 799 & 27,0 & 680 & 23,0 & 414 & 14,0 & 621 & 21,2 & \\
\hline Urbana & 1893 & 64,0 & 2159 & 73,2 & 2278 & 77,0 & 2544 & 86,0 & 2337 & 78,8 & \\
\hline Escolaridade materna (anos) & & & & & & & & & & & $<0,001$ \\
\hline$\leq 4$ & 1124 & 38,0 & 1272 & 43,0 & 887 & 30,1 & 770 & 26,0 & 680 & 23,0 & \\
\hline $5-8$ & 976 & 33,0 & 887 & 30,0 & 1065 & 36,0 & 1005 & 34,1 & 1183 & 40,6 & \\
\hline$\geq 9$ & 858 & 29,0 & 799 & 27,0 & 1006 & 34,1 & 1183 & 40,0 & 947 & 37,0 & \\
\hline Crianças < 5 anos & & & & & & & & & & & $<0,001$ \\
\hline 1 criança & 1420 & 48,4 & 1538 & 53,2 & 1864 & 63,0 & 2189 & 74,0 & 1745 & 59,0 & \\
\hline 2 crianças & 1094 & 37,0 & 1065 & 34,6 & 887 & 30,0 & 621 & 21,0 & 1006 & 34,9 & \\
\hline 3 ou mais crianças & 444 & 15,1 & 355 & 12,2 & 207 & 7,0 & 148 & 5,0 & 207 & 7,0 & \\
\hline Creche & & & & & & & & & & & $<0,001$ \\
\hline Não & 1243 & 42,0 & 1301 & 44,0 & 1059 & 35,8 & 917 & 31,0 & 769 & 26,0 & \\
\hline Sim & 1715 & 58,0 & 1657 & 56,0 & 1899 & 64,2 & 2041 & 69,0 & 2189 & 74,0 & \\
\hline $\mathrm{N}^{\circ}$ moradores & & & & & & & & & & & $<0,001$ \\
\hline $1-3$ & 562 & 19,0 & 710 & 24,0 & 769 & 26,0 & 798 & 27,0 & 740 & 25,1 & \\
\hline $4-5$ & 1272 & 43,0 & 1183 & 40,2 & 1450 & 49,0 & 1509 & 51,0 & 1449 & 49,3 & \\
\hline Mais de 6 & 1124 & 38,0 & 1069 & 36,4 & 739 & 25,0 & 651 & 22,0 & 769 & 26,0 & \\
\hline Idade materna (anos) & & & & & & & & & & & $<0,001$ \\
\hline$<21$ & 775 & 26,2 & 778 & 26,3 & 509 & 17,2 & 420 & 14,2 & 766 & 25,9 & \\
\hline $21-25$ & 735 & 24,9 & 736 & 24,9 & 787 & 26,6 & 597 & 20,2 & 790 & 26,7 & \\
\hline $26-30$ & 792 & 26,8 & 713 & 24,1 & 816 & 27,6 & 863 & 29,2 & 668 & 22,6 & \\
\hline$>30$ & 656 & 22,2 & 731 & 24,7 & 846 & 28,6 & 1078 & 36,5 & 734 & 24,8 & \\
\hline Renda & & & & & & & & & & & $<0,001$ \\
\hline $1^{\circ}$ quartil & 701 & 23,7 & 1103 & 37,3 & 393 & 13,3 & 192 & 6,5 & 376 & 12,7 & \\
\hline $2^{\circ}$ quartil & 929 & 31,4 & 837 & 28,3 & 706 & 23,9 & 547 & 18,5 & 822 & 27,8 & \\
\hline $3^{\circ}$ quartil & 648 & 21,9 & 535 & 18,1 & 813 & 27,5 & 881 & 29,8 & 778 & 26,3 & \\
\hline $4^{\circ}$ quartil & 680 & 23,0 & 483 & 16,3 & 1046 & 35,4 & 1338 & 45,2 & 982 & 33,2 & \\
\hline Etnia & & & & & & & & & & & $<0,001$ \\
\hline Branco & 807 & 27,3 & 834 & 28,2 & 1435 & 48,5 & 2256 & 76,3 & 1257 & 42,5 & \\
\hline Preto & 133 & 4,5 & 160 & 5,4 & 281 & 9,5 & 188 & 6,4 & 210 & 7,1 & \\
\hline Parda & 2018 & 68,2 & 1964 & 66,4 & 1242 & 42,0 & 514 & 17,4 & 1491 & 50,4 & \\
\hline
\end{tabular}




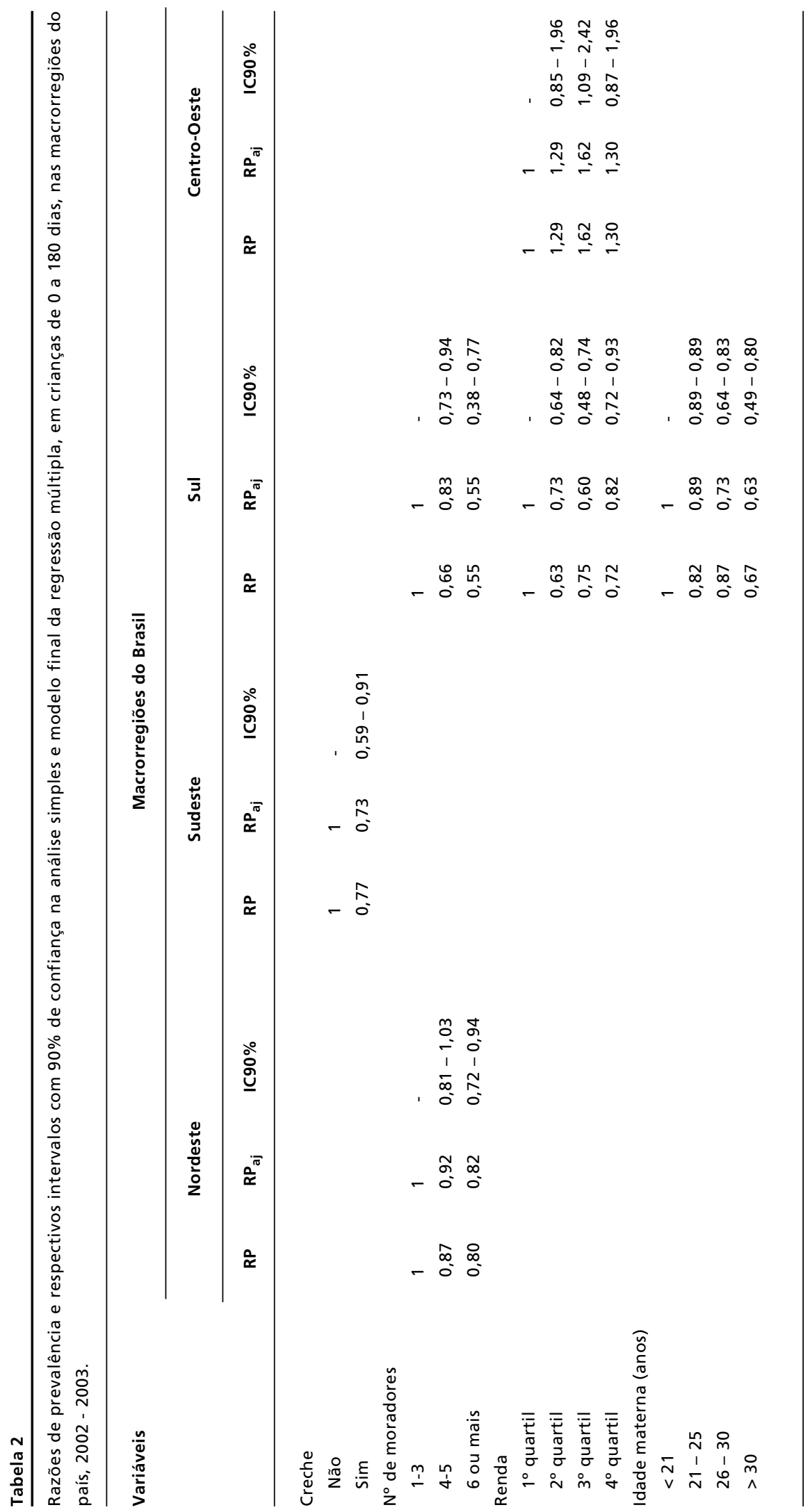




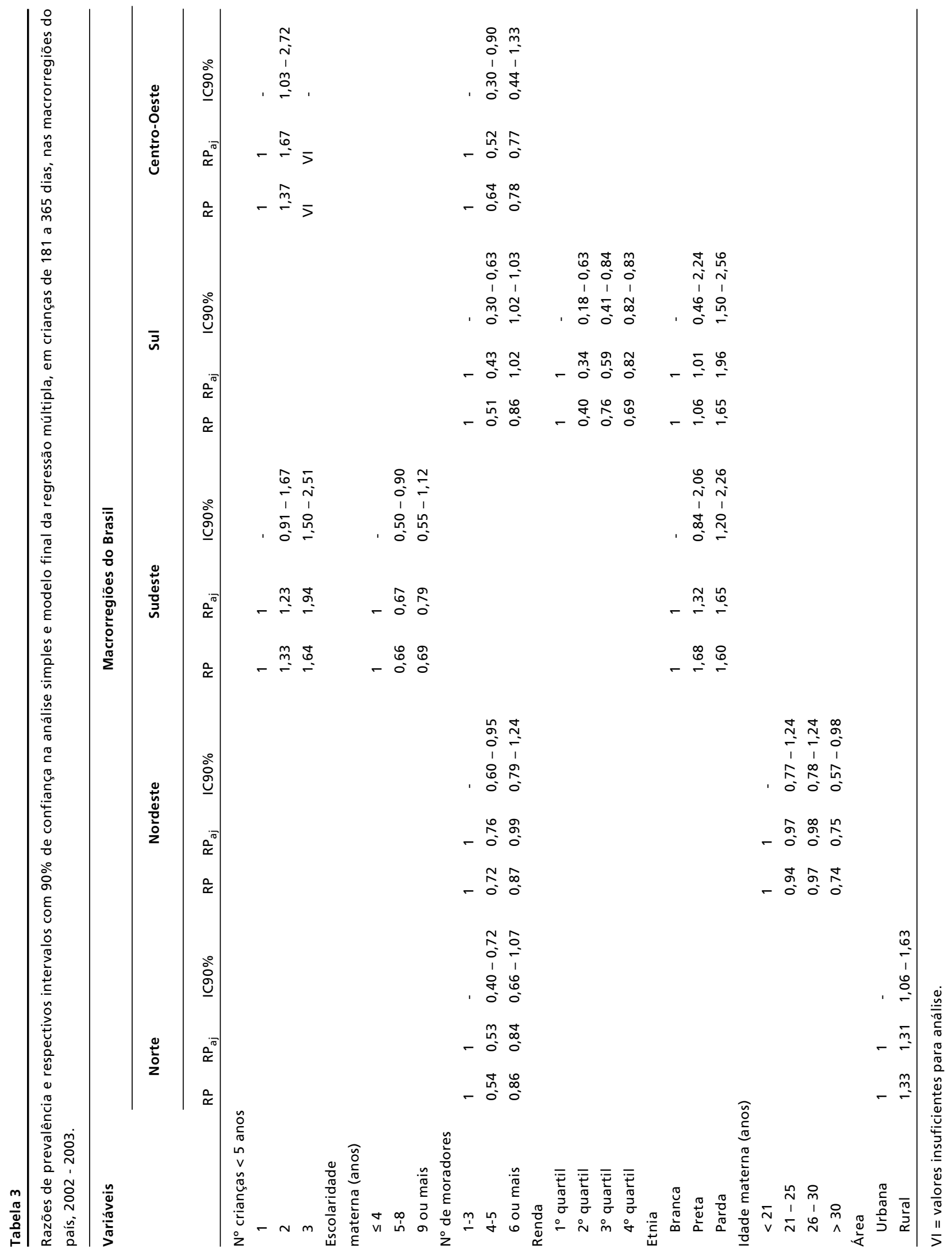




\section{Discussão}

A análise das diversidades regionais na prevalência e fatores relacionados à amamentação é importante para a definição de políticas de promoção ao aleitamento materno adequados aos diferentes cenários encontrados.

Foram identificados como fatores desfavoráveis à amamentação em todas as Regiões, a idade materna mais elevada, residir em domicílios com quatro ou mais moradores, maior renda e escolaridade materna e frequentar creches. Os fatores favoráveis à amamentação foram a presença de duas ou mais crianças menores de cinco anos na residência e cor materna preta ou parda.

As Regiões Sul e o Sudeste foram as que apresentaram número maior de fatores desfavoráveis ao aleitamento materno nas duas faixas etárias. Nessas Regiões a maior parte das mulheres tinha idade mais elevada, maior renda e cor branca, sendo tais fatores sido identificados como fatores de risco para a não amamentação. Esses resultados possivelmente se relacionam aos resultados da POF 2002/20038 e da II Pesquisa de Prevalência de Aleitamento Materno nas Capitais Brasileiras e Distrito Federal realizada pelo Ministério da Saúde em 2009,11 que constatou a baixa prevalência de aleitamento materno nessas regiões e uma explicação plausível para a menor prevalência de amamentação entre as mulheres com idade mais elevada é o fato de a maioria apresentar estabilidade profissional, que resultaria em menor tempo disponível para o cuidado do bebê, inclusive para a prática que a amamentação necessita. Outro fator que pode estar implicado é a própria decisão materna de não amamentar. Estudos realizados em Hong Kong, nos Estados Unidos e no Brasil, mostraram que mulheres com idades mais elevada apresentavam menos intenção de amamentar.11,18,19 Quanto à menor frequência de amamentação entre as mulheres de cor branca, esse dado pode ser justificado pelo maior número de populações de origem europeia, principalmente na Região Sul, que historicamente apresentam mais baixa prevalência de aleitamento materno.

Nas Regiões Sudeste e Centro-Oeste o maior número de crianças menores de cinco anos no domicílio esteve positivamente associado ao aleitamento materno. Esse resultado pode ser explicado pelo fato de mulheres multíparas já terem supostamente tido experiência anterior com a amamentação. ${ }^{20}$

Na Região Sudeste, frequentar creche esteve associada a menor prevalência de amamentação. Ainda que a situação de trabalho não tenha sido explorada na POF, pode-se supor que este resultado tenha sido influenciado pela maior inclusão das mulheres no mercado de trabalho nesta região, que torna muitas vezes necessária o uso de creche pelas crianças, dificultando a prática da amamentação e favorecendo a interrupção precoce da amamentação. Outra hipótese possível seria a falta de estrutura das creches para o acolhimento de crianças em aleitamento materno, como a falta de equipamentos para a estocagem de leite materno, ou mesmo a falta de treinamento dos funcionários das creches. ${ }^{21,22}$ Frota e Marcopito 23 concluem em seu estudo que a presença de condições que propiciam a amamentação no local de trabalho mostram que amamentar e trabalhar podem ser atividades compatíveis.

Mais de quatro moradores por domicílio foi um fator associado a menor prevalência de amamentação em quase todas as regiões do Brasil. Esse resultado pode estar possivelmente relacionado a piores condições de vida, incluindo menor nível de renda que poderia influenciar negativamente na frequência de aleitamento materno. Esses resultados concordam com os apresentados pelo "National Survey of Children's Health" (NCHS-2003), no qual se observou que famílias com mais de dois membros foi um fator relacionado a não amamentação. 24

Famílias com precárias condições de vida parecem apresentar maior chance de introduzir alimentos complementares na dieta infantil mais precocemente, devido a fatores diversos, como pressão para o retorno ao trabalho, "marketing" agressivo das indústrias de fórmulas infantis e fatores culturais que não valorizam a amamentação.24-29

Apesar da redução das diferenças na prevalência da amamentação entre áreas urbanas e rurais no Brasil, ${ }^{9}$ os dados sugerem que nas áreas rurais da Região Norte do país, a manutenção de padrões culturais ou persistência de estruturas tradicionais de apoio à família podem ter contribuído para as prevalências de aleitamento materno nas áreas rurais mais do que nas áreas urbanas. ${ }^{30}$ Vale ressaltar, no entanto, que os dados desse estudo refletem a situação observada no início da década de 2000, que podem ter sofrido mudanças nesta última década.

Neste estudo, conforme proposto, foi possível traçar um perfil dos fatores que determinam o aleitamento materno em todas as regiões do Brasil no ínicio da década anterior. As Regiões que apresentaram pior situação quanto à prevalência de amamentação foram as Regiões Sudeste e Sul, tendo sido identificados fatores possívelmente relacionados a menor frequência de amamentação.

Torna-se importante a elaboração e implemen- 
tação de políticas e programas, que guardem a especificidades das diferentes regiões do país, de modo a promover a prática da amamentação por

\section{Referências}

1. Bystrova K, Ivanova V, Edhborg M, Matthiesen AS, Ranjsö-Arvidson AB, Mukhamedrakhimov $\mathrm{R}$ Mukhamedrakhimov R, Uvnäs-Moberg K, Widström AM. Early contact versus separation: effects on mother-infant interaction one year later. Birth. 2009; 36 (2): 97-109.

2. Popkin BM, Adair L, Akin JS, Black R, Briscoe J, Flieger W. Breast-feeding and diarrheal morbidity. Pediatrics. 1990; 86 (6): 874-82.

3. Castro IRR, Montenegro Engstrom EM, Cardoso LO, Damião JJ, Rito RVFV, Gomes MASM. Tendência temporal da amamentação na cidade do Rio de Janeiro: 1996-2006. Rev Saúde Pública. 2009; 43 (6): 1021-29.

4. Grjibovski AM, Yngve A, Bygren LO, Sjöström M. Sociodemographic determinants of initiation and duration of breastfeeding in northwest Russia. Acta Paediatr. 2005; 94 (5): 588-94.

5. Fenglian X, Xiaoxian L, Colin WB, Cuiqin X, Jing W and Andy HL. A decade of change in breastfeeding in China's far north-west. Int Breastfeed J. 2006, 1: 22, 1-7.

6. Giovannini M, Banderali G, Agostoni C, Silano M, Radaelli G, Riva E. Epidemiology of breastfeeding in Italy. Acta Paediatr. Suppl 1999, 88 (430): 19-22.

7. Venâncio SI, Saldiva SRD, Monteiro CA. Tendência secular da alimentação no Brasil. Rev Saúde Pública. 2013; 47 (6) 1205-8.

8. Bocollini CS, Carvalho ML, Oliveira MIC, Vasconcelos AGG. Fatores associados à amamentação na primeira hora de vida. Rev Saúde Pública. 2011: 41 (1): 69-78.

9. Wenzel D, Souza SB. Prevalência do aleitamento materno no Brasil segundo condições socioeconômicas e demográficas. Rev Bras Crescimento Desenvolv Hum. 2011; 21 (2): 251-8

10. Brasil. Ministério da Saúde. Centro Brasileiro de Análise e Planejamento. Pesquisa nacional de demografia e saúde da criança e da mulher (PNDS): relatório final. Brasília, DF; 2008. Disponível em: http://bvsms.saude.gov.br/bvs/pnds/img/relatorio final pn ds2006.pdf

11. Venancio SI, Escuder MML, Saldiva SRD, Giugliani ERJ. Breastfeeding practice in the Brazilian capital cities and the Federal District: current status and advances. J Pediatric. 2010; 86 (4): 317-24

12. Rea MF. Avaliação das práticas diferenciais de amamentação: a questão da etnia. Rev Saúde Pública. 1994; 28 (5): 365-72.

13. Bentley ME, Dee DL, Jensen JL. Breastfeeding among low income, African-American women: power, beliefs and decision making. J Nutr. 2003; 133 (1): 3055-95.

14. Haas DM, Howard CS, Christopher M, Rowan K, Broga M, Corey $\mathrm{T}$. Assessment of breastfeeding practices and reasons for success in a Military Community Hospital. J Hum Lact. 2006; 22 (4): 439-45. períodos mais longos, visando a redução da morbidade e mortalidade no Brasil.

15. Romero SQ, Bernal R, Barbiero C, Passamonte R, Cattaneo A. A rapid ethnographic study of breastfeeding in the North and South of Italy. Int Breastfeed J. 2006; 1:14.

16. Yeoh BH, Eastwood J, Phung H, Woolfenden S. Factors influencing breastfeeding rates in south-western Sydney. J Paediatr Child Health. 2007; 43 (4): 249-55.

17. IBGE (Instituto Brasileiro de Geografia e Estatística). Pesquisa de orçamentos familiares, 2002-2003. Aquisição alimentar domiciliar per capita, Brasil e grandes regiões. Rio de Janeiro; 2004.

18. Ryan AS, Wenjun Z, Acosta A. Breastfeeding continues to increase into the new millennium. Pediatrics. 2002; 110: $1103-9$

19. Leung TF, Tam WH, Hung EC, Fok TF, Wong GW. Sociodemographic and atopic factors affecting breastfeeding intention in Chinese mothers. J Paediatr Child Health. 2003; 39 (6): 460-4.

20. Bakoula C, Veltsista A, Prezerakou A, Moustaki M, Fretzayas A, Nicolaidou P. Working mothers breastfeed babies more than housewives. Acta Paediatr. 2007; 96 (4): 510-5.

21. Ryan AS, Zhou W, Arensberg MB. The effect of employment status on breastfeeding in the United States. Women's Health Issues. 2006; 16: 243-51.

22. Zapana PM, Oliveira Mde N, Taddei JA. Factors determining the breastfeeding in children attending public and not-for-profit daycare centers in São Paulo, Brazil. Arch Latinoam Nutr. 2010; 60 (4): 360-7.

23. Frota DAL, Marcopito LF. Amamentação em mães adolescentes e não-adolescentes, Montes Claros, MG. Rev Saúde Pública 2004; 38 (1): 85-92.

24. Singh GKS, Kogan MD, Dee DL. Nativity/Immigrant Status, Race/Ethnicity, and socioeconomic determinants of breastfeeding initiation and duration in the United States 2003. Pediatrics. 2007; 119 (Suppl. 1): S38-46.

25. Victora CG, Barros FC, Halpern R, Menezes AM, Horta B, Tomasi E, Weiderpass E, Cesar JA, Olinto MT, Guimarães PRV, del Mar Garcia M, Vaughan JP. Estudo longitudinal da populaçãomaterno-infantil da região urbana do sul do Brasil, 1993: Aspectos metodológicos e resultadospreliminares. Rev Saúde Pública. 1996, 30: 34-45.

26. Rea MF, Venâncio SI, Batista LE, Santos RG, Greiner T. Possibilidades e limitações da amamentação entre mulheres trabalhadoras formais. Rev Saúde Pública. 1997; 31: 14956.

27. Vasconcelos MGL, Lira PIC, Lima MC. Duração e fatores associados ao aleitamento materno em crianças menores de 24 meses de idade no estado de Pernambuco. Rev Bras Saúde Materno Infantil. 2006; 6 (1): 99-105.

28. Oliveira LPM, Barreto MLB, Assis AMO, Braga-Junior ACR, Maria F. F. Pussik Nunes MFF, Oliveira NF, Benício MHA, Venâncio SI, Saldiva SRDM, Escuder MML. 
Preditores do retardo de crescimento linear em pré-escolares: uma abordagem multinível. Cad Saúde Pública. 2007; 23 (3): 601-13.

29. Carrascoza KC, Possobon RF, Ambrosano GMB, Costa Júnior AL, Moraes ABA. Determinantes do abandono do aleitamento materno exclusivo em crianças assistidas por programa interdisciplinar de promoção à amamentação. Ciênc Saúde Coletiva. 2011; 16 (10): 4139-46.

Recebido em 10 de dezembro de 2013

Versão final apresentada em 17 de março de 2014

Aprovado em 27 de junho de 2014
30. Shroff MR, Griffiths PL, Suchindran C, Nagalla B, Vazir S, Bentley ME. Does maternal autonomy influence feeding practices and infant growth in rural India? Soc Sci Med. 2011; 73 (3): 447-55 